\title{
Arithmetic Discrete Planes Are Quasicrystals
}

\author{
Valérie Berthé \\ LIRMM, Université Montpellier II, 161 rue Ada, 34392 Montpellier Cedex 5 - France \\ berthe@lirmm.fr
}

\begin{abstract}
Arithmetic discrete planes can be considered as liftings in the space of quasicrystals and tilings of the plane generated by a cut and project construction. We first give an overview of methods and properties that can be deduced from this viewpoint. Substitution rules are known to be an efficient construction process for tilings. We then introduce a substitution rule acting on discrete planes, which maps faces of unit cubes to unions of faces, and we discuss some applications to discrete geometry.
\end{abstract}

Keywords: digital planes; arithmetic discrete planes; tilings; word combinatorics; quasicrystals; substitutions.

\section{Introduction}

Discrete planes are known since the work of Réveillès [Rev91, see also [AAS97, to be efficiently defined in arithmetic terms, which allows a precise and effective understanding of most of their properties, see e.g. BCK07. More precisely, let $\boldsymbol{v} \in \mathbb{R}^{d}, \mu, \omega \in \mathbb{R}$. The arithmetic discrete (hyper)plane $\mathfrak{P}(\boldsymbol{v}, \mu, \omega)$ is defined as the set of points $\boldsymbol{x} \in \mathbb{Z}^{d}$ satisfying $0 \leq\langle\boldsymbol{x}, \boldsymbol{v}\rangle+\mu<\omega$. We assume now for the sake of clarity that $d=3$ but the results and methods mentioned in the present paper hold for any $d \geq 3$.

For the sake of simplicity $\boldsymbol{v}=\left(v_{1}, v_{2}, v_{3}\right)$ is assumed in all that follows to be a nonzero vector with nonnegative coordinates. We consider here integer as well as irrational parameters $\boldsymbol{v}, \mu, \omega$. We recall that the dimension of the lattice of the period vectors of an arithmetic discrete plane is equal to the dimension of the space $d$ minus the dimension of the $\mathbb{Q}$-vector space generated by the coordinates of the normal vector $\boldsymbol{v}$.

Our aim is to focus on the properties of arithmetic discrete planes obtained when noticing that they can be described thanks to a so-called cut and project construction which consists in projecting a subset of a lattice onto a plane. Indeed, arithmetic discrete planes are obtained by selecting points of the lattice $\mathbb{Z}^{3}$ : we take a slice of width $\omega$ of $\mathbb{Z}^{3}$ along the Euclidean hyperplane $P(\boldsymbol{v}, \mu)$ with equation $\langle\boldsymbol{x}, \boldsymbol{v}\rangle+\mu=0$. We thus have performed the cutting part of the construction. The interval $[0, \omega)$ is called the selection window.

Consider now the projection part. Let $\pi_{0}$ be the orthogonal projection onto the hyperplane $P_{0}$ with equation $\langle\boldsymbol{x},(1,1,1)\rangle=0$. Recall that $\boldsymbol{v}$ is assumed to have nonnegative coordinates. By projecting by $\pi_{0}$ the arithmetic discrete plane $\mathfrak{P}(\boldsymbol{v}, \mu, \omega)$, one gets a set of points of $P_{0}$ which is a Delone set, that is,

S. Brlek, C. Reutenauer, and X. Provençal (Eds.): DGCI 2009, LNCS 5810, pp. 1-12, 2009.

(C) Springer-Verlag Berlin Heidelberg 2009 
it is relatively dense (there exists $R>0$ such that any Euclidean ball of $P_{0}$ of radius $R$ contains a point of this set) and uniformly discrete (there exists $r>0$ such that any ball of radius $r$ contains at most one point of this set). We have recovered via this construction a so-called quasicrystal, that is, a discrete structure which displays long-range order without having to be periodic. The object of the present paper is to stress it. For more details, see for instance Sen95.

This selection and projection method is also widely used as an efficient method for constructing tilings. Recall that a tiling by translation of the plane by a set $T$ of (proto)tiles is a union of translates of elements of $T$ that covers the full plane, with any two tiles intersecting either on an empty set, or on a vertex or on an edge. For more details on tilings, see for instance GS87.

Note that the choice of the projection $\pi_{0}$ for the definition of the cut and project set $P_{0}$ is noncanonical. We have chosen here a projection $\pi_{0}$ that maps in a one-to-one way our selected set of points to a discrete set endowed with an underlying lattice (see Section 2.1), whereas the orthogonal projection of this set onto the line orthogonal to $P_{0}$ is dense in the selection window $[0, \omega)$ when $\boldsymbol{v}$ has irrational entries. We will use this latter denseness result in Section 2.3. Such a choice for $\pi_{0}$ enters the framework of cut and project schemes. For precise definitions, see BM2000.

This paper is organized as follows. Section 2 introduces a tiling and a twodimensional coding associated with arithmetic discrete planes. We will show how to deduce properties of configurations and regularity properties such as repetitivity. The idea will be to associate with finite configurations occuring in the discrete plane subintervals of the selection window $[0, \omega)$. This allows us to handle simultaneously arithmetic discrete planes having the same normal vector. We then want to "compare" discrete planes having different normal vectors. We thus introduce in Section 3 a substitution rule acting on discrete planes, and whose action is described with respect to their normal vector $\boldsymbol{v}$. We conclude this survey of applications of tiling theory in the study of arithmetic discrete planes by focusing in Section 4 on so-called boundary words.

\section{Discrete Planes Coded as Multidimensional Words}

Let $\mathfrak{P}(\boldsymbol{v}, \mu, \omega)$ be an arithmetic discrete plane. We have obtained so far a discrete set of points $\pi_{0}(\mathfrak{P}(\boldsymbol{v}, \mu, \omega))$ of the plane $P_{0}$ which has a priori no specific algebraic structure. Neverthless, we have obtained this set via a selection process which involves the lattice $\mathbb{Z}^{3}$ and a projection $\pi_{0}$ which also displays some kind of regularity. We first show how to recover a lattice underlying the arithmetic discrete plane $\mathfrak{P}(\boldsymbol{v}, \mu, \omega)$. We assume that we are in the standard case $\omega=\|\boldsymbol{v}\|_{1}$.

\subsection{A Tiling by Lozenges}

One way to recover this lattice is to associate with the quasicrystal $\pi_{0}(\mathfrak{P}(\boldsymbol{v}, \mu, \omega))$ a tiling of the plane $P_{0}$ obtained by connecting with edges points of the quasicrystal. One checks that one gets a tiling of the plane $P_{0}$ by three kinds of tiles, 
namely the three regular lozenges being the projections by $\pi_{0}$ of the three faces of the unit cube depicted on Fig. 1 below. We call them $T_{i}$, for $i=1,2,3$. We will denote this tiling by $T(\boldsymbol{v}, \mu, \omega)$.
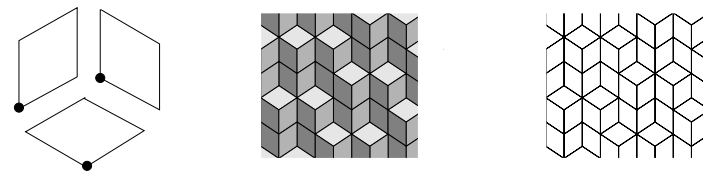

Fig. 1. Left: The three lozenge tiles $T_{1}, T_{2}, T_{3}$. Middle and Right: From the stepped plane $\mathfrak{P}\left(\boldsymbol{v}, \mu,\|\boldsymbol{v}\|_{1}\right)$ to the tiling $T\left(\boldsymbol{v}, \mu,\|\boldsymbol{v}\|_{1}\right)$.

We also associate with $\mathfrak{P}(\boldsymbol{v}, \mu, \omega)$ a surface $\mathcal{P}(\boldsymbol{v}, \mu, \omega)$ in $\mathbb{R}^{3}$ called stepped plane defined as the union of translates of faces of the unit cube whose vertices belong to $\mathfrak{P}(\boldsymbol{v}, \mu, \omega)$. The projection by $\pi_{0}$ of $\mathcal{P}(\boldsymbol{v}, \mu, \omega)$ yields the tiling $T(\boldsymbol{v}, \mu, \omega)$ (see Fig. 1).

Conversely, any tiling made of the three lozenge tiles $T_{i}$, for $i=1,2,3$, admits a unique lifting as a surface in $\mathbb{R}^{3}$ up to translation by the vector $(1,1,1)$, with this lifting being equal to $\mathcal{P}(\boldsymbol{v}, \mu, \omega)$ if the tiling equals $T(\boldsymbol{v}, \mu, \omega)$. The idea of the proof is to associate with every vertex of the tiling a height function that is uniquely determined and whose definition is globally consistent. For more details, see [Thu89] and for a proof in this context, see ABFJ07. Tilings by the three tiles $T_{i}$ are widely studied in the framework of dimers on the honeycomb graph (see [KO05).

We now pick for each tile $T_{i}$ a particular vertex, called its distinguished vertex. More precisely, let $\left(\boldsymbol{e}_{1}, \boldsymbol{e}_{2}, \boldsymbol{e}_{3}\right)$ stand for the canonical basis of $\mathbb{R}^{3}$. We consider the following faces of the unit cube: $F_{1}=\left\{\lambda e_{2}+\mu e_{3} \mid 0 \leq \lambda, \mu \leq 1\right\}, F_{2}=$ $\left\{-\lambda e_{1}+\mu e_{3} \mid 0 \leq \lambda, \mu \leq 1\right\}, F_{3}=\left\{-\lambda e_{1}-\mu e_{2} \mid 0 \leq \lambda, \mu \leq 1\right\}$. One has $T_{i}=\pi_{0}\left(F_{i}\right)$, for $i=1,2,3$. For $\boldsymbol{x} \in \mathbb{Z}^{3}$, the distinguished vertex of $\boldsymbol{x}+F_{i}$ is then defined as $\boldsymbol{x}$, and similarly the distinguished vertex of the tile $\boldsymbol{y}+T_{i}$ of the tiling $T(\boldsymbol{v}, \mu, \omega)$ is defined as $\boldsymbol{y}$.

Note that one has a one-to-one correspondence between tiles $T_{i}+\boldsymbol{y}$ of the tiling $T(\boldsymbol{v}, \mu, \omega)$ and faces $F_{i}+\boldsymbol{x}$ in $\mathbb{R}^{3}$ of the stepped plane $(\boldsymbol{v}, \mu, \omega)$ : indeed, for any tile $T_{i}+\boldsymbol{y}$ of the tiling $T(\boldsymbol{v}, \mu, \omega)$, there exists a unique $\boldsymbol{x}$ such that $\pi_{0}(\boldsymbol{x})=\boldsymbol{y}$ and $0 \leq\langle\boldsymbol{x}, \boldsymbol{v}\rangle+\mu<\|\boldsymbol{v}\|_{1}$.

Now one checks that the set of distinguished vertices of $\mathfrak{P}(\boldsymbol{v}, \mu, \omega)$ is a lattice (see [BV00]). We thus have found a lattice underlying the arithmetic discrete plane $\mathfrak{P}\left(\boldsymbol{v}, \mu,\|\boldsymbol{v}\|_{1}\right)$ even if the coordinates of $\boldsymbol{v}$ are rationally independent, that is, even if the arithmetic discrete plane has no nonzero period vector. Since the set of distinguished vertices is a lattice that can be assimilated to $\mathbb{Z}^{2}$, we can code as a $\mathbb{Z}^{2}$-word over the alphabet $\{1,2,3\}$ any arithmetic discrete plane. See [BV00] for more details and Fig. 2 below for an illustration. 

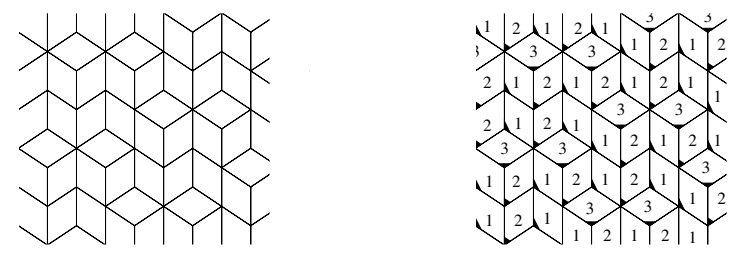

Fig. 2. From a discrete plane to its coding as a two-dimensional word

\subsection{Configurations}

We want now to localize with respect to the value $\langle\boldsymbol{x}, \boldsymbol{v}\rangle$ in the selection window $[0, \omega)$ the distinguished vertices of faces of a given type. Recall that we work in the standard case. One has $0 \leq\langle\boldsymbol{x}, \boldsymbol{v}\rangle+\mu<\|\boldsymbol{v}\|_{1}=v_{1}+v_{2}+v_{3}$.

Assume first that $0 \leq\langle\boldsymbol{x}, \boldsymbol{v}\rangle+\mu<v_{1}$. Then $\boldsymbol{x}+\boldsymbol{e}_{2}, \boldsymbol{x}+\boldsymbol{e}_{3}, \boldsymbol{x}+\boldsymbol{e}_{2}+\boldsymbol{e}_{3}$ all belong to $\mathfrak{P}(\boldsymbol{v}, \mu, \omega)$. We thus deduce that the full face $F_{1}+\boldsymbol{x}$ is included in $\mathcal{P}(\boldsymbol{v}, \mu, \omega)$.

Similarly, assume $v_{1} \leq\langle\boldsymbol{x}, \boldsymbol{v}\rangle+\mu<v_{1}+v_{2}$ (respectively $v_{1}+v_{2} \leq\langle\boldsymbol{x}, \boldsymbol{v}\rangle+\mu<$ $v_{1}+v_{2}+v_{3}$ ). Then $\boldsymbol{x}-\boldsymbol{e}_{1}, \boldsymbol{x}+\boldsymbol{e}_{3}, \boldsymbol{x}-\boldsymbol{e}_{1}+\boldsymbol{e}_{3}$ (respectively $\boldsymbol{x}-\boldsymbol{e}_{1}, \boldsymbol{x}-\boldsymbol{e}_{2}$, $\left.\boldsymbol{x}-\boldsymbol{e}_{1}-\boldsymbol{e}_{2}\right)$ all belong to $\mathfrak{P}(\boldsymbol{v}, \mu, \omega)$. We thus deduce that the full face $F_{2}+\boldsymbol{x}$ (respectively $\left.F_{3}+\boldsymbol{x}\right)$ is included in $\mathcal{P}(\boldsymbol{v}, \mu, \omega)$.

In fact more can be said. One checks for $\boldsymbol{x} \in \mathbb{Z}^{3}$ that

$$
\boldsymbol{x}+F_{i} \subset \mathcal{P}(\boldsymbol{v}, \mu, \omega) \text { if and only if } \sum_{k<i} v_{k} \leq\langle\boldsymbol{x}, \boldsymbol{v}\rangle+\mu<\sum_{k \leq i} v_{k} .
$$

Note that we have only sketched the proof of the "if " direction. For a full proof and more details, see [BV00].

In other words, our convention for the choice of a distinguished vertex of a face implies that a face of type $i$ with distinguished vertex $\boldsymbol{x}$ is included in $\mathcal{P}(\boldsymbol{v}, \mu, \omega)$ if and only if $\langle\boldsymbol{x}, \boldsymbol{v}\rangle+\mu \in I_{i}$. Hence, we have cut the selection interval $\left[0,\|\boldsymbol{v}\|_{1}\right)$ into three subintervals,

$$
I_{1}=\left[0, v_{1}\right), I_{2}=\left[v_{1}, v_{1}+v_{2}\right), I_{3}=\left[v_{1}+v_{2}, v_{1}+v_{2}+v_{3}\right),
$$

each of them corresponding to the occurrences of the distinguished vertex of a particular type of face.

This holds not only for faces but also for finite unions of faces. A configuration of the tiling $T(\boldsymbol{v}, \mu, \omega)$ is an edge-connected finite union of lozenge tiles contained in the tiling. We asume that $\mathbf{0}$ is always a distinguished vertex of one of the faces of a configuration. We then consider occurrences of configurations up to translation. Note that liftings in $\mathcal{P}(\boldsymbol{v}, \mu, \omega)$ of configurations correspond to usual local configurations of discrete planes. By abuse of terminology, we also call them here configurations. The configuration $C$ is said to occur at $\boldsymbol{y}$ in the tiling 
$T(\boldsymbol{v}, \mu, \omega)$ if $C+\boldsymbol{y}$ is included in it. We then can associate with the configuration $C$ the set $I_{C}$ defined as the closure of the set

$$
\left\{\langle\boldsymbol{x}, \boldsymbol{v}\rangle+\mu \mid \boldsymbol{y}=\pi_{0}(\boldsymbol{x}), \boldsymbol{x} \in \mathfrak{P}(\boldsymbol{v}, \mu, \omega), C \text { occurs in } T(\boldsymbol{v}, \mu, \omega) \text { at } \boldsymbol{y}\right\} .
$$

One checks that the set $I_{C}$ is an interval if the dimension of the $\mathbb{Q}$-vector space generated by the coordinates of $\boldsymbol{v}$ is at least 2. We use the denseness in the acceptance window $[0, \omega)$ of $(\langle\boldsymbol{x}, \boldsymbol{v}\rangle)_{\boldsymbol{x} \in \mathbb{Z}^{2}}$. If $\boldsymbol{v}$ has integer coprime entries and $\mu$ is also an integer, $I_{C}$ is a set of consecutive integers. We use in this latter case Bezout's lemma. For a proof, see BV00. It also uses the fact that $I_{C}$ is described as an intersection of intervals which are preimages of the intervals $I_{i}$ under the action of the translations $x \mapsto x+v_{j}$, for $i, j \in\{1,2,3\}$. We thus have here again divided the selection window $[0, \omega)$ into unions of intervals associated with configurations.

Let us illustrate it on one example. Consider the configuration $C=T_{1} \cup\left(T_{1}+\right.$ $\left.\boldsymbol{e}_{3}\right)$. Configuration $C$ occurs at $\boldsymbol{y}$ if and only if $\langle\boldsymbol{x}, \boldsymbol{v}\rangle+\mu \in I_{1}$ and $\left\langle\boldsymbol{x}+\boldsymbol{e}_{3}, \boldsymbol{v}\right\rangle+\mu=$ $\langle\boldsymbol{x}, \boldsymbol{v}\rangle+v_{3}+\mu \in I_{1}$, that is, $\langle\boldsymbol{x}, \boldsymbol{v}\rangle+\mu \in I_{1} \cap\left(I_{1}-v_{3}\right)$. Hence $I_{C} \neq \emptyset$ if and only if $v_{1}>v_{3}$. If $v_{1}>v_{3}$, then $I_{C}=\left[0, v_{1}-v_{3}\right)$.

Note that this approach still holds when one works with arithmetic discrete planes with general width $[0, \omega)$. One can similarly associate with a configuration $C$ a set $I_{C}$ defined here again as an intersection of intervals themselves intersected with $[0, \omega)$.

\subsection{Applications}

We thus have been able to associate with a configuration $C$ an interval $I_{C}$ of the selection window $[0, \omega)$. Let us discuss several properties that can be deduced from this correspondence.

A configuration $C$ occurs if and only if $I_{C}$ is nonempty. Let us come back to the example of the previous section. The lifting $F_{1} \cup\left(F_{1}+\boldsymbol{e}_{3}\right)$ of the configuration $C$ occurs up to translation in $\mathcal{P}(\boldsymbol{v}, \mu, \omega)$ if and only if $I_{C}=\left[0, v_{1}-v_{3}\right)$ is nonempty, that is, $v_{1}>v_{3}$. Note that this does not depend on the parameter $\mu$.

We thus recover an effective way to check whether a configuration occurs in a given arithmetic discrete plane. Indeed, the set $I_{C}$ can be effectively determined such as shown in Section 2.2. It thus remains to check whether the coresponding intersection is nonempty.

More generally, we deduce that two discrete planes with the same normal vector and same width have the same configurations. Indeed, intervals $I_{C}$ do not depend on $\mu$ and only depend on $\boldsymbol{v}$. We furthermore can count the number of configurations of a given size and shape. Indeed we have to determine the bounds of the intervals $I_{C}$ and then count them. We can also determine the frequencies of occurrence of configurations: they are given by the lengths of the intervals $I_{C}$. In this latter case, we use not only denseness results but equidistribution results. For more details, see e.g. BV00] and BFJP07]. Note that these methods hold for general widths $\omega$. 
These methods are classic in word combinatorics, symbolic dynamics, or tiling theory. Let us recall that standard and naive arithmetic discrete lines are coded thanks to the Freeman code as Sturmian words [Lot02, PF02].

Consider now repetitivity results. The radius of a configuration is defined as the minimal radius of a disk containing this configuration. Two configurations in the plane $P_{0}$ are said identical if they only differ by a translation vector. A tiling is said repetitive if for every configuration $C$ of radius $r$ there exists a positive number $R$ such that every configuration of radius $R$ contains $C$. This is a counterpart of the notion of uniform recurrence in word combinatorics and symbolic dynamics. In other words, configurations appear "with bounded gaps". Repetitive tilings can be considered as ordered structures.

Let $C$ be a given configuration that occurs in the tiling $T$. We consider the interval associated with $C$. Repetitivity can then be deduced from the following fact. Given any interval $I$ of $\mathbb{R} / \mathbb{Z}$, the sequence $(n \alpha)_{n \in \mathbb{N}}$ enters the interval $I$ with bounded gaps, that is, there exists $N \in \mathbb{N}$ such that any sequence of $N$ successive values of the sequence contains a value in $I$ [Sla67]. We apply it to the interval $I_{C}$ and work modulo $\omega$ in $\mathbb{R} / \omega \mathbb{Z}$. We thus deduce that the configuration $C$ occurs in the tiling $T$ with bounded gaps.

\section{Substitutions}

We have been so far able to describe properties of arithmetic discrete planes sharing the same normal vector $\boldsymbol{v}$. We now want to be able to relate two discrete planes with different normal vectors $\boldsymbol{v}$ and $\boldsymbol{v}^{\prime}$. We focus on the case $\boldsymbol{v}=\boldsymbol{M \boldsymbol { v } ^ { \prime }}$, where $M$ is a 3 by 3 square matrix with entries in $\mathbb{N}$ having determinant equal to 1 or -1 (such a matrix is called unimodular).

\subsection{Multidimensional Continued Fractions}

Our motivation relies on the fact that unimodular transformations are the basic steps when expanding vectors under the action of a unimodular multidimensional continued fraction algorithm, such as Jacobi-Perron or Brun algorithms (here we expand a normal vector $\boldsymbol{v}$ of a plane). It is well known that arithmetic discrete lines and their codings as Sturmian words are perfectly well described by Euclid's algorithm and by the continued fraction expansion of their slope [Lot02, PF02]. We want to generalize this to the higher-dimensional case.

It is also well-known that there exists no canonical multidimensional continued fraction algorithm. We focus here on unimodular algorithms such as described in Bre81, Sch00]. Such multidimensional continued fraction algorithms produce sequences of matrices in $G L(d, \mathbb{Z})$ as follows.

Let $X \subset \mathbb{R}^{d}$. A $d$-dimensional continued fraction map over $X$ is a map $T$ : $X \rightarrow X$ such that $T(X) \subset X$ and, for any $\boldsymbol{x} \in X$, there is a matrix in $G L(d, \mathbb{Z})$ depending on $\boldsymbol{x}$ (we thus denote it by $M(\boldsymbol{x})$ ) satisfying: $\boldsymbol{x}=M(\boldsymbol{x}) \cdot T(\boldsymbol{x})$. The associated continued fraction algorithm consists in iteratively applying the map $T$ on a vector $\boldsymbol{x} \in X$. This yields the following sequence of matrices, called 
the continued fraction expansion of $\boldsymbol{x}:\left(M\left(T^{n}(\boldsymbol{x})\right)\right)_{n \in \mathbb{N}}$. If the matrices have nonnegative entries, the algorithm is said nonnegative.

Consider for instance the Jacobi-Perron algorithm. Its projective version is defined on the unit square $[0,1) \times[0,1)$ by:

$$
(\alpha, \beta) \mapsto\left(\frac{\beta}{\alpha}-\left\lfloor\frac{\beta}{\alpha}\right\rfloor, \frac{1}{\alpha}-\left\lfloor\frac{1}{\alpha}\right\rfloor\right)=(\{\beta / \alpha\},\{1 / \alpha\}) .
$$

Its linear version is defined on the positive cone $X=\left\{(a, b, c) \in \mathbb{R}^{3} \mid 0 \leq a, b<c\right\}$ by:

$$
T(a, b, c)=(b-\lfloor b / a\rfloor a, c-\lfloor c / a\rfloor a, a) .
$$

We set $\left(a_{0}, b_{0}, c_{0}\right):=(a, b, c)$ and $\left(a_{n+1}, b_{n+1}, c_{n+1}\right):=T^{n}\left(a_{n}, b_{n}, c_{n}\right)$, for $n \in \mathbb{N}$. Let $B_{n+1}=\left\lfloor b_{n} / a_{n}\right\rfloor a_{n}, C_{n}=\left\lfloor c_{n} / a_{n}\right\rfloor$. One has

$$
\left(\begin{array}{l}
a_{n} \\
b_{n} \\
c_{n}
\end{array}\right)=\left(\begin{array}{lll}
0 & 0 & 1 \\
1 & 0 & B_{n+1} \\
0 & 1 & C_{n+1}
\end{array}\right)\left(\begin{array}{l}
a_{n+1} \\
b_{n+1} \\
c_{n+1}
\end{array}\right) .
$$

The Jacobi-Perron algorithm is thus a unimodular nonnegative continued fraction algorithm. With the previous notation, $M(\boldsymbol{x})=M_{B_{1}, C_{1}}$ for $\boldsymbol{x}=\left(a_{0}, b_{0}, c_{0}\right)$, by setting $M_{B, C}:=\left(\begin{array}{lll}0 & 0 & 1 \\ 1 & 0 & B \\ 0 & 1 & C\end{array}\right)$ for $B, C \in \mathbb{N}$.

Note that the sequence of admissible Jacobi-Perron digits $\left(B_{n}, C_{n}\right)_{n \geq 1}$ satisfies $0 \leq B_{n} \leq C_{n}$ and $B_{n}=C_{n}$ implies $B_{n+1} \geq 1$ for all $n$.

The idea is now to consider the expansion of a given normal vector $\boldsymbol{v} \in X$. Let $\boldsymbol{v}^{(n)}$ stand for $T^{n}(\boldsymbol{v})$. One expands $\boldsymbol{v}$ as

$$
\boldsymbol{v}=M_{B_{1}, C_{1}} \cdots M_{B_{n}, C_{n}} \boldsymbol{v}^{(n)} .
$$

\subsection{Substitution Rules}

Let us come back to our geometric framework. We fix a matrix $M \in S L(3, \mathbb{N})$ (for instance $M=M_{B, C}$ ). We assume furthermore that we are in the standard case $\omega=\|\boldsymbol{v}\|_{1}$. We want to find an algorithmic way to go from $\mathcal{P}_{M \boldsymbol{v}, \mu,\|M \boldsymbol{v}\|_{1}}$ to $\mathcal{P}_{\boldsymbol{v}, \mu,\|\boldsymbol{v}\|_{1}}$.

The idea is to use the fact that

$$
\langle\boldsymbol{x}, M \boldsymbol{v}\rangle=\left\langle{ }^{t} M \boldsymbol{x}, \boldsymbol{v}\right\rangle \text { for any } \boldsymbol{x} \in \mathbb{Z}^{3} .
$$

Furthermore, for computational reasons, we change our convention for faces. We now define faces as follows: $E_{1}=\left\{\lambda e_{2}+\mu e_{3} \mid 0 \leq \lambda, \mu \leq 1\right\}, E_{2}=\left\{\lambda e_{1}+\mu e_{3} \mid\right.$ $0 \leq \lambda, \mu \leq 1\}, E_{3}=\left\{\lambda e_{1}+\mu e_{2} \mid 0 \leq \lambda, \mu \leq 1\right\}$. We loose the notion of distinguished vertex but we obtain here a characterization of faces that are contained in $\mathcal{P}\left(\boldsymbol{v}, \mu,\|\boldsymbol{v}\|_{1}\right)$ that will be here easier to handle than (11): the face $\boldsymbol{y}+E_{j}$ is a subset of $\mathcal{P}\left(\boldsymbol{v}, \mu,\|\boldsymbol{v}\|_{1}\right)$ if and only if

$$
0 \leq\langle\boldsymbol{y}, \boldsymbol{v}\rangle+\mu<v_{j}=\left\langle\boldsymbol{e}_{j}, \boldsymbol{v}\right\rangle .
$$


Let us assume now that the face $\boldsymbol{y}+F_{j}$ is a subset of $\mathcal{P}\left(M \boldsymbol{v}, \mu,\|M \boldsymbol{v}\|_{1}\right)$. One has

$$
0 \leq\langle\boldsymbol{y}, M \boldsymbol{v}\rangle+\mu=\left\langle{ }^{t} M \boldsymbol{y}, \boldsymbol{v}\right\rangle+\mu\left\langle\left\langle\boldsymbol{e}_{j}, M \boldsymbol{v}\right\rangle=\left\langle{ }^{t} M \boldsymbol{e}_{j}, \boldsymbol{v}\right\rangle .\right.
$$

The interval $\left[0,\left\langle{ }^{t} M \boldsymbol{e}_{j}, \boldsymbol{v}\right\rangle\right)$ is nonempty according to the assumptions made on $M$. Since $M$ has nonegative entries, we can divide the interval $\left[0,\left\langle{ }^{t} M \boldsymbol{e}_{j}, \boldsymbol{v}\right\rangle\right)$ into subintervals of respective lengths $\left\langle\boldsymbol{e}_{i}, \boldsymbol{v}\right\rangle=v_{i}$, for $i=1,2,3$. We know that the point $\left\langle{ }^{t} M \boldsymbol{y}, \boldsymbol{v}\right\rangle+\mu$ belongs to one of these intervals. Hence there exist a vector $\boldsymbol{S}$ and $i \in\{1,2,3\}$ such that

$$
0 \leq\left\langle{ }^{t} M \boldsymbol{y}, \boldsymbol{v}\right\rangle+\mu-\langle\boldsymbol{S}, \boldsymbol{v}\rangle\left\langle v_{i},\right.
$$

which implies that the face ${ }^{t} \boldsymbol{M} \boldsymbol{y}-\boldsymbol{S}+E_{i}$ is a subset of $\mathcal{P}\left(\boldsymbol{v}, \mu,\left\|\boldsymbol{v}_{1}\right\|\right)$.

We thus have associated with a face $\boldsymbol{y}+E_{j}$ of $\mathcal{P}\left(M \boldsymbol{v}, \mu,\|M \boldsymbol{v}\|_{1}\right)$ a face $\boldsymbol{x}+E_{i}$ where $\boldsymbol{x}={ }^{t} M \boldsymbol{y}-\boldsymbol{S}$ in $\mathcal{P}\left(\boldsymbol{v}, \mu,\|\boldsymbol{v}\|_{1}\right)$, and the entry $m_{j i}$ of $M$ satisfies $m_{j i} \neq 0$.

One checks that this map is onto (we use the fact that $M$ is an invertible matrix as a matrix acting on $\mathbb{Z}^{3}$ ) but not one-to-one. This is why we will work below (see Eq. (3) ) with the inverse of this map.

\subsection{Substitutions in Word Combinatorics}

Let us formalize this map. In particular, we need to define more precisely the way we divide the interval $\left[0,\left\langle{ }^{t} M \boldsymbol{e}_{j}, \boldsymbol{v}\right\rangle\right)$ into subintervals of respective lengths $\left\langle\boldsymbol{e}_{i}, \boldsymbol{v}\right\rangle=v_{i}$. In other words, we have to choose a way of tiling the larger interval by these smaller intervals. For that purpose, we first recall basic definitions from word combinatorics. For more details, see Lot02, PF02.

We consider the set of finite words over the alphabet $\{1,2,3\}$. We denote this set as $\{1,2,3\}^{*}$. Endowed with the concatenation, it is a free monoid. A substitution is a morphism of the free monoid. A substitution $\sigma$ thus replaces letters by words, and moreover, since it is a morphism, it satisfies $\sigma(u v)=$ $\sigma(u) \sigma(v)$, for any $u, v$ words in $\{1,2,3\}^{*}$. Consider for example the substitution $\sigma$ defined by $\sigma(1)=12, \sigma(2)=13, \sigma(3)=1$. The incidence matrix $M_{\sigma}$ of $\sigma$ is defined as the 3 by 3 square matrix with entry $(i, j)$ entry equal to the number of occurrences of the letter $i$ in $\sigma(j)$. With our example, $M_{\sigma}=\left[\begin{array}{lll}1 & 1 & 1 \\ 1 & 0 & 0 \\ 0 & 1 & 0\end{array}\right]$. A substitution is said unimodular if the determinant of its incidence matrix equals 1 or -1 . Lastly, let $\boldsymbol{l}:\{1,2,3\}^{*} \rightarrow \mathbb{N}^{3}$ be the abelianization map $\boldsymbol{l}(w)=$ ${ }^{t}\left(|w|_{1},|w|_{2},|w|_{3}\right)$.

We now can introduce the notion of generalized substitution, such as introduced in AI02. Let $\sigma$ be a unimodular substitution. The map $E_{1}^{*}(\sigma)$ maps faces on unions of faces. It is defined respectively on faces $\boldsymbol{x}+E_{i}$ and on sets of faces $G, H$ as follows

$$
\begin{gathered}
E_{1}^{*}(\sigma)\left(\boldsymbol{x}+E_{i}\right)=\sum_{j \in\{1,2,3\}} \sum_{P, \sigma(j)=P i S}\left(M_{\sigma}^{-1}(\boldsymbol{x}+\boldsymbol{l}(S))+E_{j}\right), \\
E_{1}^{*}(G \cup H)=E_{1}^{*}(G) \cup E_{1}^{*}(H) .
\end{gathered}
$$


Let us explain the notation " $P, \sigma(j)=P i S$ " used above. We consider letters $j$ such that $\sigma(j)$ contains $i$ and make the summation on all occurrences of the letter $i$ in $\sigma(j)$. We thus decompose $\sigma(j)$ as $P i S$, where $P, S$ are words in $\{1,2,3\}^{*}$ and we "label" each occurrence of $i$ by the prefix $P$ that precedes it. One of the main interests of this kind of geometric replacement map is that it maps stepped planes onto stepped planes:

Theorem 1. AI02, Fer06] Let $\sigma$ be a unimodular substitution. Let $\boldsymbol{v} \in \mathbb{R}_{+}^{d}$ be a positive vector. The generalized substitution $E_{1}^{*}(\sigma)$ maps without overlaps the stepped plane $\mathcal{P}_{\boldsymbol{v}, \mu,\|\boldsymbol{v}\|_{1}}$ onto $\mathcal{P}_{t} M_{\sigma} \boldsymbol{v}, \mu,\left\|{ }^{t} M_{\sigma} \boldsymbol{v}\right\|_{1}$.

The proof of the theorem is based on the ideas sketched above in Section 3.2 Note that if $\boldsymbol{y}=M_{\sigma}^{-1}(\boldsymbol{x}+\boldsymbol{l}(S))$, then $\boldsymbol{x}=M_{\sigma} \boldsymbol{y}-\boldsymbol{l}(S)$. We recover the expression obtained in Section 3.2 .

\subsection{Back to Discrete Planes}

Let us see now how to use Theorem 1 in discrete geometry.

We first start with the question of the generation of discrete planes. We illustrate our strategy with the Jacobi-Perron algorithm. We also fix $\mu=0$. We use the notation $\mathcal{P}(\boldsymbol{v})$ for $\mathcal{P}\left(\boldsymbol{v}, 0,\|\boldsymbol{v}\|_{1}\right)$. We associate with the matrix $M_{B, C}$ the substitution $\sigma_{B, C}: 1 \mapsto 1,2 \mapsto 1^{B} 3,3 \mapsto 1^{C} 2$ having the transpose of $M_{B, C}$ as incidence matrix. Let $\boldsymbol{v} \in X$. By Theorem [1] one has

$$
E_{1}^{*}\left(\sigma_{B, C}\right)(\mathcal{P}(T(\boldsymbol{v}))=\mathcal{P}(\boldsymbol{v}) .
$$

Assume now we are given the sequence of Jacobi-Perron digits $\left(B_{n}, C_{n}\right)_{n \geq 1}$ produced by the Jacobi-Perron expansion of the vector $\boldsymbol{v}$. One has

$$
E_{1}^{*}\left(\sigma_{\left(B_{1}, C_{1}\right)}\right) \circ E_{1}^{*}\left(\sigma_{\left(B_{2}, C_{2}\right)}\right) \cdots \circ E_{1}^{*}\left(\sigma_{\left(B_{n}, C_{n}\right)}\right)\left(\mathcal{P}\left(\boldsymbol{v}^{(n)}\right)=\mathcal{P}(\boldsymbol{v}) .\right.
$$

Note that the lower unit cube $\mathcal{U}:=E_{1}+E_{2}+E_{3}$ belongs to every arithmetic discrete plane with parameter $\mu=0$. We deduce that

$$
E_{1}^{*}\left(\sigma_{\left(B_{1}, C_{1}\right)}\right) \circ \cdots \circ E_{1}^{*}\left(\sigma_{\left(B_{n}, C_{n}\right)}\right)(\mathcal{U}) \subset \mathcal{P}(\boldsymbol{v})
$$

The question is now whether the patterns $E_{1}^{*}\left(\sigma_{\left(B_{1}, C_{1}\right)}\right) \circ \cdots \circ E_{1}^{*}\left(\sigma_{\left(B_{n}, C_{n}\right)}\right)(\mathcal{U})$ generate the whole plane $\mathcal{P}(\boldsymbol{v})$, that is, whether

$$
\lim _{n \rightarrow \infty} E_{1}^{*}\left(\sigma_{\left(B_{1}, C_{1}\right)}\right) \circ \cdots \circ E_{1}^{*}\left(\sigma_{\left(B_{n}, C_{n}\right)}\right)(\mathcal{U})=\mathcal{P}(\boldsymbol{v})
$$

If yes, then we get a simple algorithmic generation process of the arithmetic discrete plane $\mathcal{P}(\boldsymbol{v})$. This is illustrated in Fig. 3 below.

This question has been answered in the Jacobi-Perron case in [I093. Indeed, it has been proved that if there exists no integer $n$ such that for all $k$

$$
B_{n+3 k}=C_{n+3 k}, C_{n+3 k+1}-B_{n+3 k+1} \geq 1, B_{n+3 k+1} \neq 0, B_{n+3 k+2}=0,
$$




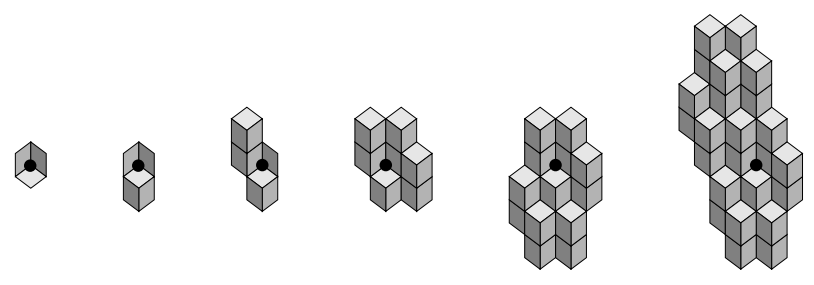

Fig. 3. Some iterations of a generalized substitution acting on the lower unit cube $\mathcal{U}$

the sequence of patterns $E_{1}^{*}\left(\sigma_{\left(B_{1}, C_{1}\right)}\right) \circ \cdots \circ E_{1}^{*}\left(\sigma_{\left(B_{n}, C_{n}\right)}\right)(\mathcal{U})$ generates the whole plane $\mathcal{P}(\boldsymbol{v})$. Otherwise, it is not possible to generate the whole plane by starting only from $\mathcal{U}$, but there exists a finite set of faces $\mathcal{V}$ with $\mathcal{U} \subset \mathcal{V}$ such that the whole plane is generated starting from $\mathcal{V}$ instead of $\mathcal{U}$, that is, the sequence of patterns $E_{1}^{*}\left(\sigma_{\left(B_{1}, C_{1}\right)}\right) \circ \cdots \circ E_{1}^{*}\left(\sigma_{\left(B_{n}, C_{n}\right)}\right)(\mathcal{V})$ generates the whole plane $\mathcal{P}(\boldsymbol{v})$.

Let us stress that we are not only able to substitute, i.e., to replace faces by unions of faces, but also to desubstitute, i.e., to perform the converse operation, by using the algebraic property $E_{1}^{*}(\sigma)^{-1}=E_{1}^{*}\left(\sigma^{-1}\right)$ (where $\sigma$ is considered as a morphism of the free group is an automorphism). For more details, see [BF09] in the case of Brun algorithm. This idea is also used in [Fer09, still for Brun algorithm, where algorithms are given for the digital plane recognition and digital plane generation problems.

Let us quote a further classical question in the study of discrete planes that can be handled under the formalism of generalized substitutions $E_{1}^{*}(\sigma)$. The question is to find the smallest width $\omega$ for which the plane $\mathcal{P}_{\boldsymbol{v}, \mu, \omega}$ is connected (either edge connected or vertex connected). The case of rational parameters has been solved in [JT09a]. For the case of irrational parameters, see DJT09. The method used in both papers relies on the use of a particular unimodular multidimensional continued fraction algorithm (the so-called fully subtractive algorithm) and on the use of Eq. (2).

\section{Conclusion}

We have discussed here several ideas concerning the study of arithmetic discrete planes inspired by tiling theory and word combinatorics. We have focused on a multidimensional coding (via faces) of configurations and discrete planes. We can also associate with configurations of discrete planes a boundary word coding as a finite word their boundary. The use of word combinatorics and the study of boundary words has recently proven to be particularly useful in discrete geometry. Let us also quote in particular BLPR08, which gives a nice characterization of digitally convex polyominoes in terms of the Lyndon decomposition of the word coding their boundary. See also BKP09] which gives an efficient recognition algorithm of nonintersecting paths based on the use of suffix trees.

Boundary words also allow us to describe the topology of the configurations of discrete planes we generate via the method described in Section 3.4 Let us 
recall the following result of [Ei03. Let $\sigma$ be an invertible three-letter substitution (by invertible, we mean that $\sigma$ considered as a morphism of the free group is an automorphism). The boundary word of $E_{1}^{*}(\sigma)(\mathcal{U})$ is produced by the mirror image of the inverse of $\sigma$. We thus can apply this result to get a description of the boundary of the generating patterns BLPP09]. Note that these patterns can be described in terms of pseudo-squares and pseudo-hexagons such as introduced in BN91 which gives a characterization of polyominoes which tile the plane. See also [BFP09] for corresponding efficient recognition algorithms and BBGL09. for a study of a family of so-called double pseudo-squares.

\section{Acknowledgements}

We would like to thank Geneviève Paquin and Xavier Provençal for their very useful comments.

\section{References}

[AAS97] Andres, É., Acharya, R., Sibata, C.: The Discrete Analytical Hyperplanes. Graph. Models Image Process. 59, 302-309 (1997)

[AI02] Arnoux, P., Ito, S.: Pisot substitutions and Rauzy fractals. Bull. Bel. Math. Soc. Simon Stevin 8, 181-207 (2001)

[ABI02] Arnoux, P., Berthé, V., Ito, S.: Discrete planes, $\mathbb{Z}^{2}$-actions, Jacobi-Perron algorithm and substitutions. Ann. Inst. Fourier (Grenoble) 52, 1001-1045 (2002)

[ABFJ07] Arnoux, P., Berthé, V., Fernique, T., Jamet, D.: Functional stepped surfaces, flips and generalized substitutions. Theoret. Comput. Sci. 380, 251-267 (2007)

[BN91] Beauquier, D., Nivat, M.: On translating one polyomino to tile the plane. Discrete Comput. Geom. 6, 575-592 (1991)

[BF09] Berthé, V., Fernique., T.: Brun expansions of stepped surfaces (Preprint) [BV00] Berthé, V., Vuillon, L.: Tilings and Rotations on the Torus: A TwoDimensional Generalization of Sturmian Sequences. Discrete Math. 223, 27-53 (2000)

[BFJP07] Berthé, V., Fiorio, C., Jamet, D., Philippe, F.: On some applications of generalized functionality for arithmetic discrete planes. Image and Vision Computing 25, 1671-1684 (2007)

[BLPP09] Berthé, V., Lacasse, A., Paquin, G., Provençal, X.: Boundary words for arithmetic discrete planes generated by Jacobi-Perron algorithm (Preprint)

[BBGL09] Blondin Massé, A., Brlek, S., Garon, A., Labbé, S.: Christoffel and Fibonacci Tiles. In: Brlek, S., Reutenauer, C., Provençal, X. (eds.) DGCI 2009. LNCS, vol. 5810, pp. 68-79. Springer, Heidelberg (2009)

[BFP09] Brlek, S., Fédou, J.M., Provençal, X.: On the tiling by translation problem. Discrete Applied Mathematics 157, 464-475 (2009)

[BKP09] Brlek, S., Koskas, M., Provençal, X.: A linear time and space algorithm for detecting path intersection. In: Brlek, S., Reutenauer, C., Provençal, X. (eds.) DGCI 2009. LNCS, vol. 5810, pp. 398-409. Springer, Heidelberg (2009) 
[BLPR08] Brlek, S., Lachaud, J.O., Provençal, X., Reutenauer, C.: Lyndon + Christoffel $=$ Digitally Convex. Pattern Recognition 42, 2239-2246 (2009)

[Bre81] Brentjes, A.J.: Mathematical Centre Tracts. Multi-dimensional continued fraction algorithms 145, Matematisch Centrum, Amsterdam (1981)

[BCK07] Brimkov, V., Coeurjolly, D., Klette, R.: Digital Planarity - A Review. Discr. Appl. Math. 155, 468-495 (2007)

[BM2000] Baake, M., Moody, R.V., Robert, V. (eds.): Directions in mathematical quasicrystals. CRM Monograph Series, vol. 13. American Mathematical Society, Providence (2000)

[DJT09] Domenjoud, E., Jamet, D., Toutant, J.-L.: On the connecting thickness of arithmetical discrete planes. In: Brlek, S., Reutenauer, C., Provençal, X. (eds.) DGCI 2009. LNCS, vol. 5810, pp. 362-372. Springer, Heidelberg (2009)

[Ei03] Ei, H.: Some properties of invertible substitutions of rank d and higher dimensional substitutions. Osaka J. Math. 40, 543-562 (2003)

[Fer06] Fernique, T.: Multi-dimensional Sequences and Generalized Substitutions. Int. J. Fond. Comput. Sci. 17, 575-600 (2006)

[Fer09] Fernique, T.: Generation and recognition of digital planes using multidimensional continued fractions. Pattern Recognition 432, 2229-2238 (2009)

[GS87] Grunbaum, B., Shepard, G.: Tilings and patterns. Freeman, New-York (1987)

[I093] Ito, S., Ohtsuki, M.: Modified Jacobi-Perron algorithm and generating Markov partitions for special hyperbolic toral automorphisms. Tokyo J. Math. 16, 441-472 (1993)

[JT09a] Jamet, D., Toutant, J.-L.: Minimal arithmetic thickness connecting discrete planes. Discrete Applied Mathematics 157, 500-509 (2009)

[KO05] Kenyon, R., Okounkov, O.: What is a dimer? Notices Amer. Math. Soc. 52, 342-343 (2005)

[Lot02] Lothaire, N.: Algebraic combinatorics on words. Cambridge University Press, Cambridge (2002)

[PF02] Pytheas Fogg, N.: Substitutions in Dynamics, Arithmetics, and Combinatorics. In: Berthé, V., Ferenczi, S., Mauduit, C., Siegel, A. (eds.) Frontiers of Combining System. Lecture Notes in Mathematics, vol. 1794, Springer, Heidelberg (2002)

[Rev91] Reveillès, J.-P.: Calcul en Nombres Entiers et Algorithmique. Thèse d'état, Université Louis Pasteur, Strasbourg, France (1991)

[Sch00] Schweiger, F.: Multi-dimensional continued fractions. Oxford Science Publications, Oxford Univ. Press, Oxford (2000)

[Sen95] Senechal, M.: Quasicrystals and geometry. Cambridge University Press, Cambridge (1995)

[Sla67] Slater, N.B.: Gaps and steps for the sequence $n \theta \bmod 1$. Proc. Cambridge Phil. Soc. 63, 1115-1123 (1967)

[Thu89] Thurston, W.P.: Groups, tilings and finite state automata. In: AMS Colloquium lectures. Lectures notes distributed in conjunction with the Colloquium Series (1989) 